\title{
Preischemic treadmill exercise improves short-term memory by inhibiting hypoperfusion-induced disruption of blood-brain barrier after bilateral common carotid arteries occlusion
}

\author{
Jae-Min Lee', Seung-Soo Baek', Tae-Woon Kim² ${ }^{2}$ Hye-Sang Park², Sang-Seo Park², Jong-Min Park ${ }^{3}$, Youn-Jung Kim³ , Hyun-Seob Lee4, \\ Mal-Soon Shin ${ }^{5, *}$
}

'Department of Sport \& Health Science, College of Art \& Culture, Sangmyung University, Seoul, Korea

2Department of Physiology, College of Medicine, Kyung Hee University, Seoul, Korea

${ }^{3}$ College of Nursing Science, Kyung Hee University, Seoul, Korea

${ }^{4}$ Department of Physical Education, Korea University, Seoul, Korea

${ }^{5}$ School of Global Sport Studies, Korea University, Sejong, Korea

Bilateral common carotid arteries occlusion (BCCAO) causes an abrupt reduction of cerebral blood flow, and this method has been used to investigate the effects of chronic cerebral hypoperfusion on vascular dementia and neuronal injuries. Chronic cerebral hypoperfusion leads to functional changes in the hippocampus and then results in a cognitive impairment. We investigated the effect of preischemic treadmill exercise on short-term memory and blood-brain barrier integration following cerebral hypoperfusion caused by BCCAO. The rats in the preischemic treadmill exercise and $\mathrm{BCCAO}$ group were made to run on a treadmill for 30 min once a day for 4 weeks. At 4 weeks after performing treadmill exercise, right carotid artery was ligated, and 1 week after, left common carotid artery was ligated. At 20 days after BCCA0, short-term memory was evaluated. Half of the rats were sacrificed 2 days after BCCAO and the other rats were sacrificed at 3 weeks after BCCAO. Immunohistochemistry and western blot were performed. Preischemic treadmill exercise alleviated impairment of short-term memory in the step-down avoidance task. Preischemic treadmill exercise reduced microvascular injury in the hippocampus. Preischemic treadmill exercise prevented the reduction of zonula occludens- 1 in the hippocampus and inhibited the activation of matrix metalloproteinase-9. Therefore, pre-conditioning treadmill exercise might be used as a therapeutic strategy for the prevention of stroke in patients.

Keywords: Preischemic treadmill exercise, Cerebral hypoperfusion, Bilateral common carotid artery occlusion, Short-term memory

\section{INTRODUCTION}

Cerebrovascular disease is a common cause of cognitive impairment and dementia, and this disease contributes to cognitive decline in the neurodegenerative dementia (O'Brien et al., 2003). There is a correlation between the severity of memory dysfunction and the decline of cerebral blood flow (Snyder et al., 2015). Bilateral common carotid arteries occlusion (BCCAO) causes an abrupt reduction of cerebral blood flow, and this method has been used to

investigate the effects of chronic cerebral hypoperfusion on vascular dementia and neuronal injuries (Farkas et al., 2007). Chronic cerebral hypoperfusion leads to functional changes in the hippocampus and then results in a cognitive impairment (Farkas et al., 2007). BCCAO model showed short-term and long-term memory impairments in the Morris water maze test, eight-arm radial maze test, and passive avoidance test (Kumaran et al., 2008; Lee et al., 2017; Zhang et al., 2011).

The blood-brain barrier (BBB) is formed by the endothelial
${ }^{*}$ Corresponding author: Mal-Soon Shin (iD https://orcid.org/0000-0002-7462-2211 School of Global Sport Studies, Korea University, 2511 Sejong-ro, Sejong 30019, Korea

E-mail:malsoon@korea.ac.kr

Received: April 30, 2019 / Accepted: May 30, 2019
This is an Open Access article distributed under the terms of the Creative Commons Attribution Non-Commercial License (http://creativecommons.org/licenses/by-nc/4.0/) which permits unrestricted non-commercial use, distribution, and reproduction in any medium, provided the original work is properly cited. 
cells of cerebral microvessels, providing an interface between the peripheral circulation and the central nervous system. The BBB is composed of brain capillary endothelial cells with pericytes, the basal lamina, and end-feet of astrocytes. In the endothelial cells, zonula occludens (ZO) family and the actin cytoskeleton are involved in regulation of cytoplasmic tight junction integrity. ZO-1 is a well-known tight junction protein and ZO-1 reflects the severity of pathological change of BBB. Chronic hypoxic hypoperfusion decreases integrity of tight junction in the BBB, and then induces increased paracellular permeability, directly contributing to cerebral vasogenic edema, hemorrhagic transformation, and increased mortality (Sandoval and Witt, 2008). Under ischemic insults, the expressions of ZO-1 and occludin in the ischemic penumbra were decreased (Xia et al., 2013).

Matrix metalloproteinases (MMPs) are extracellular proteolytic enzymes. MMPs digest the endothelial basal lamina and play an important role in degradation of the BBB. MMP-9 level in the BBB was upregulated after onset of focal cerebral ischemia, in contrast, MMP-9 knock-out mice were protected against ischemic and traumatic brain injury (Asahi et al., 2001). Tissue plasminogen activator (tPA) upregulated brain MMP-9 level in ischemic stroke, suggesting that combination therapy targeting MMPs may improve tPA therapy (Tsuji et al., 2005). Impaired tight junction proteins, ZO-1, claudin-5, and occludin, increased BBB permeability with up-regulation of MMP-2 and MMP-9 (Feng et al., 2011).

Physical exercise is known to exerts protective effect on neuronal injury and improve memory in neurodegenerative diseases (Lee et al., 2017; Lee et al., 2018a; Sim et al., 2004). However, many questions have been raised as to whether exercise before onset of cerebral stoke is also effective. Preischemic exercise ameliorated BBB dysfunction after stroke by reducing MMP-9 overexpression strengthening basal lamina (Guo et al., 2008). Cechetti et al. (2012) examined the effects of three different exercise protocols (preischemia exercise, postischemia exercise, and preischemia with postischemia exercise) against BCCAO-induced cognitive deficit. This cognitive deficit was reversed in the rats performing postischemia exercise and in the rats performing pre-ischemia with postischemia exercise (Cechetti et al., 2012). Preischemic treadmill exercise also exerted neuroprotective effect against transient cerebral ischemia by inhibiting apoptosis in the hippocampus (Aboutaleb et al., 2015). In the present study, we investigated the effect of preischemic treadmill exercise on short-term memory and BBB integration following cerebral hypoperfusion caused by BCCAO.

\section{MATERIALS AND METHODS}

\section{Experimental animals}

Male Wistar rats ( $80 \pm 10 \mathrm{~g}, 4$ weeks old) were used in this experiment. The experimental procedures were performed in accordance with the animal care guidelines of the National Institutes of Health and the Korean Academy of Medical Sciences. The rats were randomly divided into three groups ( $\mathrm{n}=20$ in each group): the sham group, the BCCAO group, and the preischemic treadmill exercise and BCCAO group.

\section{Treadmill exercise protocol}

The rats in the preischemic treadmill exercise and BCCAO group were made to run on a treadmill for 30 min once a day for 4 weeks starting 4 weeks after birth, according to the previously described method (Aboutaleb et al., 2015). The treadmill exercise load consisted of running at $2 \mathrm{~m} / \mathrm{min}$ for the first of $5 \mathrm{~min}$, at $3 \mathrm{~m} /$ $\mathrm{min}$ for the next $5 \mathrm{~min}$, and then at $5 \mathrm{~m} / \mathrm{min}$ for the last $20 \mathrm{~min}$ at 0 degree of inclination.

\section{BCCAO}

At 4 weeks after performing treadmill exercise, the rats were anesthetized by subcutaneous injection of $200 \mathrm{mg} / \mathrm{kg}$ body weight Zoletil 50 (Virbac Laboratories, Carros, France). A right ventral incision in the neck was performed to expose the right common carotid artery. The right carotid artery was double ligated with 3-0 silk (Ailee, Seoul, Korea) just below the carotid bifurcation. The left common carotid artery occlusion was performed in the same way at one week after right common carotid artery occlusion. Sham animals underwent the same operation without arterial ligation.

\section{Step-down avoidance test}

At 20 days after BCCAO, short-term memory was evaluated using the step-down avoidance test (Cho et al., 2018). The rats were positioned on a $7 \times 25-\mathrm{cm}$ platform, at the height of $2.5 \mathrm{~cm}$, and allowed to rest on the platform for $2 \mathrm{~min}$. The platform faced a $42 \times 25$-cm grid of parallel $0.1-\mathrm{cm}$ caliber stainless steel bars, which were spaced $1 \mathrm{~cm}$ apart. In the training session, the animals received a $0.5-\mathrm{mA}$ scramble foot shock for $3 \mathrm{sec}$ immediately upon stepping down. The retention time was assessed $24 \mathrm{hr}$ after the training session. The time between being placed on the platform and stepping down and placing all four paws on the grid, was defined as the latency in the step-down avoidance task. If this latency was over $300 \mathrm{sec}$, it was recorded as $300 \mathrm{sec}$. 


\section{Immunohistochemistry}

Half of the rats were sacrificed 2 days after BCCAO and the other rats were sacrificed at 3 weeks after BCCAO. Immunohistochemistry was performed, in a similar way to the previous method (Moon et al., 2018). Serial coronal sections of $40-\mu \mathrm{m}$ thickness were obtained using a freezing microtome (Leica, Nussloch, Germany). For the visualization of rat endothelial cells antigen-1 (RECA1) immunohistochemistry was performed. After being blocked with $10 \%$ normal rabbit serum for $1 \mathrm{hr}$, the sections were incubated overnight at $4^{\circ} \mathrm{C}$ with RECA1 antibody $(1: 1,000 ; \mathrm{Ab}$ cam, Cambridge, UK). The sections were then incubated for $2 \mathrm{hr}$ with the biotinylated rat secondary antibody (1:200; Vector Laboratories, Burlingame, CA, USA). The bound secondary antibody was then amplified using a Vector Elite ABC kit (Vector Laboratories) for $1 \mathrm{hr}$ at room temperature. For staining, the sections were incubated in a solution consisting of $0.02 \% \mathrm{DAB}$ and $0.03 \%$ $\mathrm{H}_{2} \mathrm{O}_{2}$ in $50 \mathrm{mM}$ Tris- $\mathrm{HCl}(\mathrm{pH}, 7.6)$ for approximately $5 \mathrm{~min}$, following which they were washed with PBS and mounted onto gelatin-coated slides. Cover slips were mounted using Permount (Thermo Fisher Scientific Inc., Waltham, MA, USA). The number of positive cells per section was counted in 5 random fields from every specimen with a Nikon Eclipse 80i microscope (magnification, Nikon Corp., Tokyo, Japan).

\section{Western blot analysis}

Half of the rats were sacrificed 2 days after BCCAO and the other rats were sacrificed at 3 weeks after BCCAO. Western blotting was performed, in a similar way to the previous method (Cho et al., 2018; Moon et al., 2018). Tissue samples harvested from the hippocampus were lysed in the protein lysis buffer containing $50 \mathrm{mM}$ Tris-HCI $(\mathrm{pH}, 7.5), 150 \mathrm{mM} \mathrm{NaCl}, 0.5 \%$ deoxycholic acid, $1 \%$ nonidet-P40 (NP40), $0.1 \%$ sodium dodecyl sulfate (SDS), $1 \mathrm{mM}$ phenylmethylsulfonyl fluoride, and $100-\mu \mathrm{m} / \mathrm{mL}$ leupeptin. Protein concentration was measured using a colorimetric protein assay kit (Bio-Rad, Hercules, CA, USA). Protein of $30 \mu \mathrm{g}$ was separated on SDS-polyacrylamide gels and transferred onto a nitrocellulose membrane (Schleicher \& Schuell GmbH, Dassel, Germany). The membranes were blocked in $5 \%$ nonfat skim milk in TBST for $1 \mathrm{hr}$ at room temperature followed by incubation with antibodies against ZO-1 (1:50; LifeSpan BioSciences, Inc., Seattle, WA, USA) and MMP-9 (1:1,000; Bioworld Technology, St. Louis Park, MN, USA). The primary antibodies were detected with horseradish peroxidase-conjugated secondary antibody (1:2,000; Santa Cruz Biotechnology Inc., Santa Cruz, CA, USA). Band detection was performed using an enhanced ECL detection system (Amersham Pharmacia Biotechnology GmbH, Freiburg, Germany). To compare the relative expressions of proteins, the detected bands were calculated densitometrically using Image-Pro Plus software (Media Cybernetics Inc., Silver Spring, MD, USA).

\section{Statistical analysis}

The results are expressed as mean \pm standard error of the mean. IBM SPSS Statistics ver. 23.0 (IBM Co., Armonk, NY, USA) was used for statistical analysis. For the comparison among the groups, One-way analysis of variance and Duncan post hoc test were performed with $P<0.05$ as an indication of statistical significance.

\section{RESULTS}

\section{Preischemic treadmill exercise alleviates short-term} memory impairment after BCCAO

Short-term memory was measured using the step-down avoidance test (Fig. 1) Latency time in the BCCAO group was shorter compared to the sham group. In contrast, treadmill exercise increased latency time in the BCCAO group.

\section{Preischemic treadmill exercise prevents damage to} microvessels in the hippocampal CA1 region after BCCAO

Photomicrographs of RECA1-positive microvessels in the hippocampal CA1 region are presented in Fig. 2. We found that the mean perimeter of the microvessels in the hippocampal CA1 region at $48 \mathrm{hr}$ after BCCAO did not differ. However, at 3 weeks postinjury, the mean perimeter of the microvessels in the hippo-

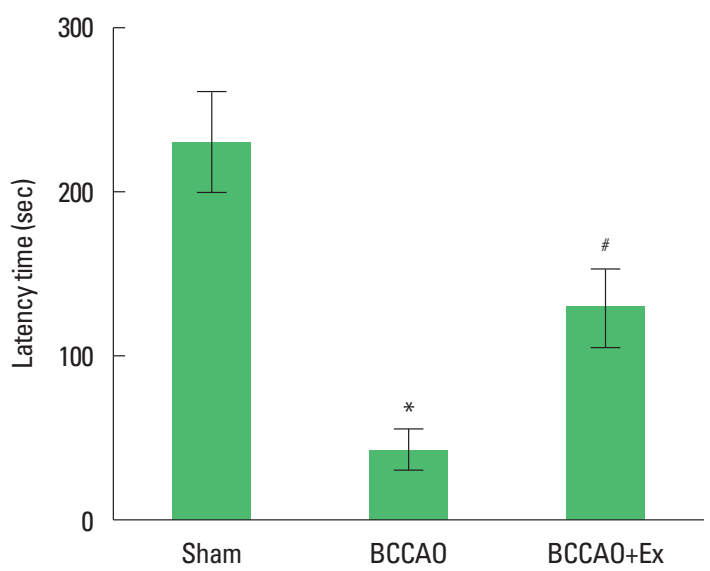

Fig. 1. Treadmill exercise alleviates shot-term memory impairment after bilateral common carotid artery occlusion (BCCAO) in the step-down avoidance task. ${ }^{*} P<0.05$ compared with the sham group. ${ }^{\sharp} P<0.05$ compared with the BCCAO group. 

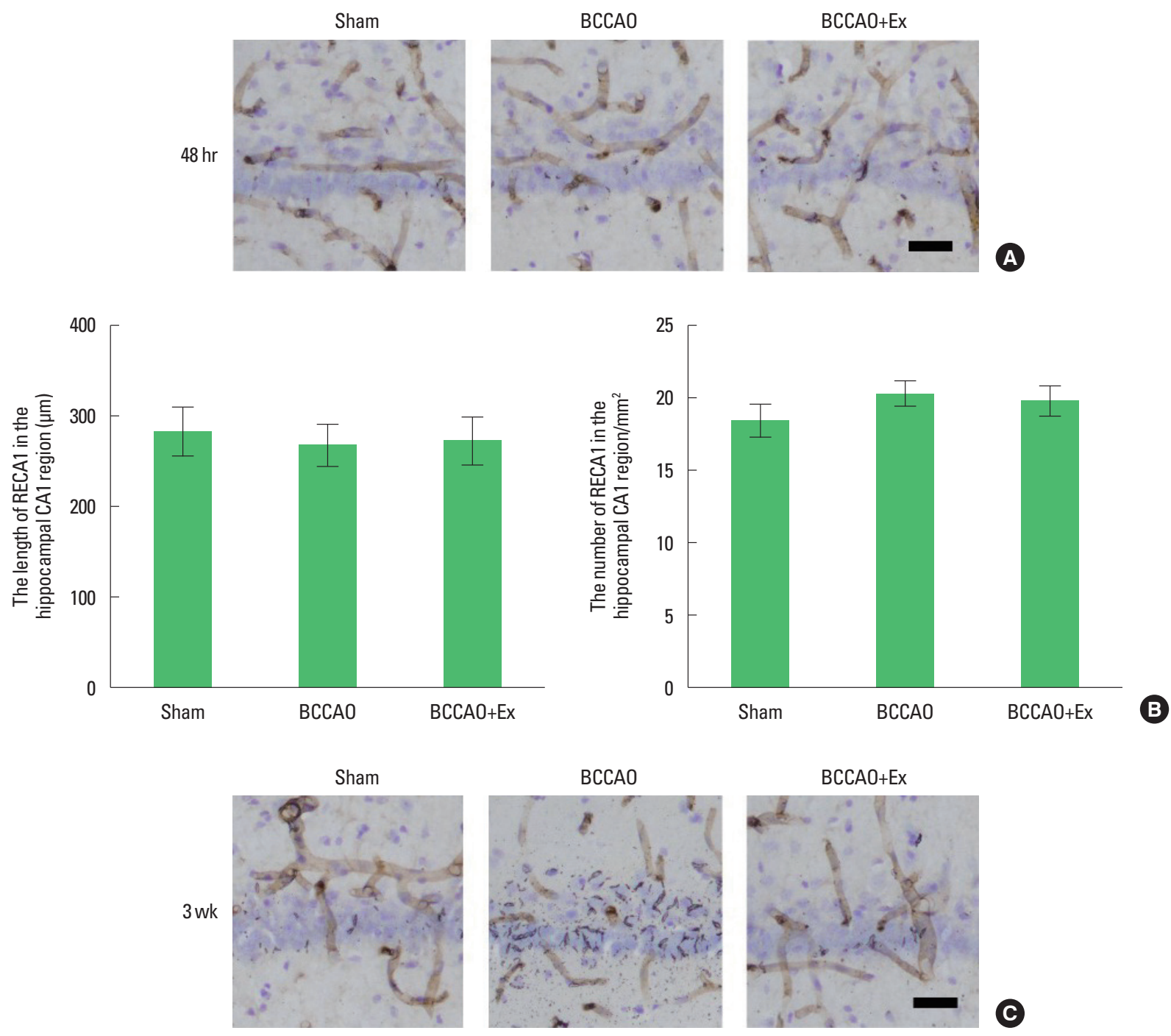

c
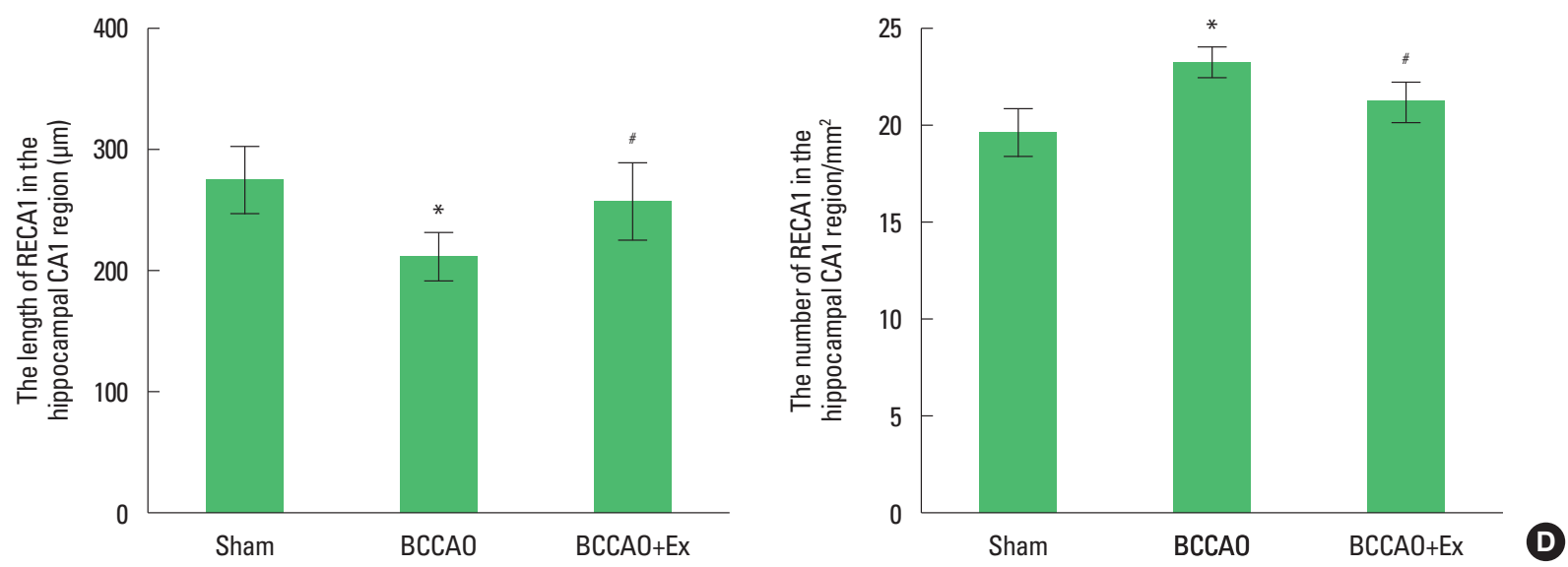

Fig. 2. Treadmill exercise prevents damage to microvessels in the hippocampal CA1 region after bilateral common carotid artery occlusion (BCCAO). (A) Photomicrographs of rat endothelial cells antigen-1 (RECA1)-positive microvessels in the hippocampal CA1 region after $48 \mathrm{hr}$. (B) The length and number of RECA1 after $48 \mathrm{hr}$. (C) Photomicrographs of RECA1-positive microvessels in the hippocampal CA1 region after 3 weeks. (D) The length and number of RECA1 after 3 weeks. Scale bar represents $50 \mu \mathrm{m}$. ${ }^{*} P<0.05$ compared with the sham group. ${ }^{*} P<0.05$ compared with the BCCAO group. 

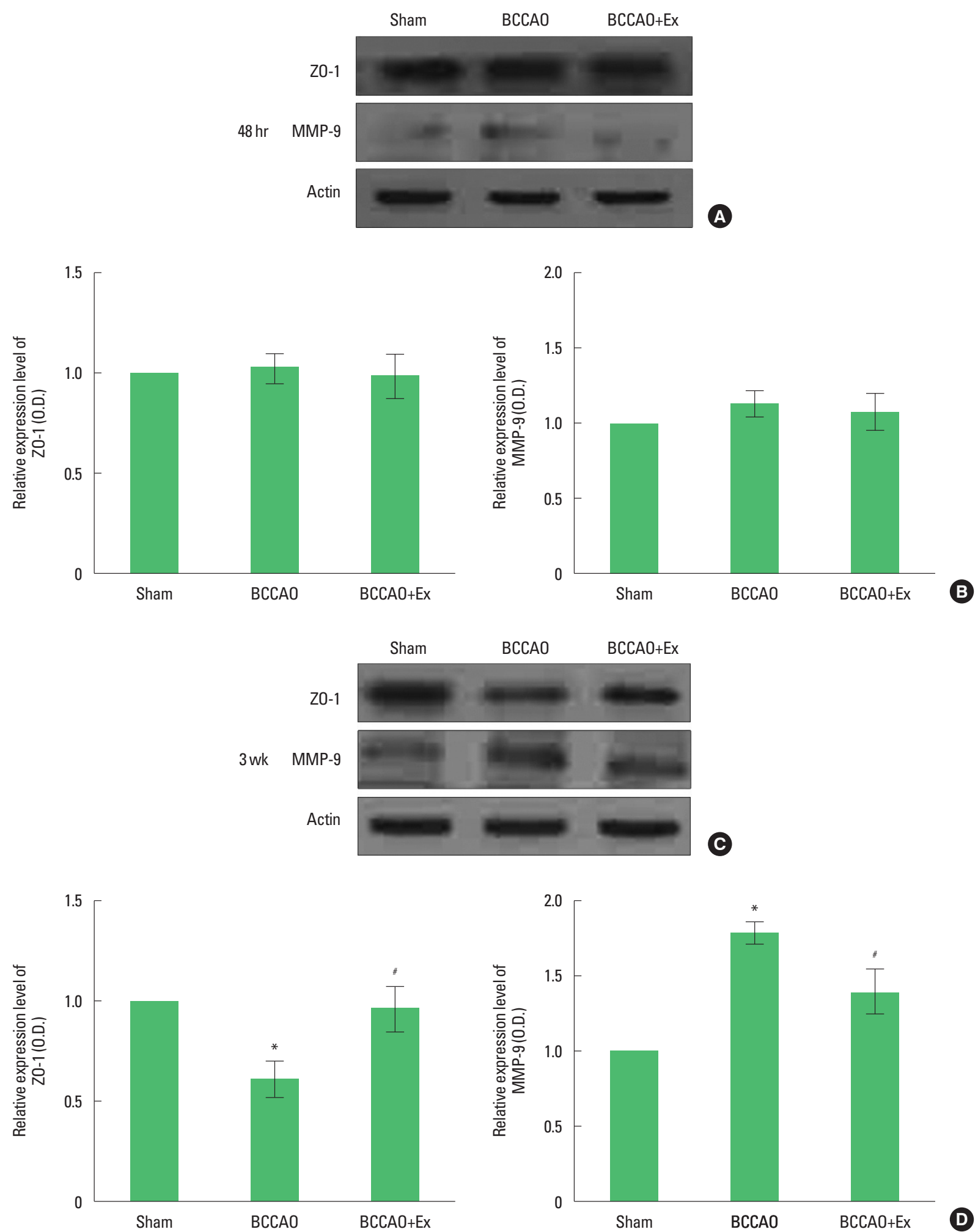

Fig. 3. Treadmill exercise prevents the loss of tight junction and activation of matrix metalloproteinase (MMP)-9 in the hippocampus after bilateral common carotid artery occlusion (BCCAO). (A) Representative bands of zonula occludens (ZO)-1 and MMP-9 in the hippocampus after $48 \mathrm{hr}$. (B) Relative expressions ZO-1 and MMP-9 after $48 \mathrm{hr}$. (C) Representative bands ZO-1 and MMP-9 in the hippocampus after 3 weeks. (D) Relative expressions ZO-1 and MMP-9 after 3 weeks. ${ }^{*} P<0.05$ compared with the sham group. ${ }^{*} P<0.05$ compared with the BCCAO group. 
campal CA1 region was short length and sections were narrow and constricted diameters. In contrast, treadmill exercise alleviated microvessels damage in the hippocampal CA1 region in the BCCAO group.

\section{Preischemic treadmill exercise prevents the loss of tight junction and inhibits activation of MMP-9 in the hippocampus after BCCAO}

Presentative western blots and quantitative data are shown in Fig. 3. We found that the level of ZO-1 in the hippocampal CA1 region at $48 \mathrm{hr}$ after BCCAO did not differ. However, at 3 weeks postinjury, the level of ZO-1 in the hippocampal CA1 region was decreased after BCCAO. In contrast, treadmill exercise increased the expression of ZO-1 in the hippocampal CA1 region in the $\mathrm{BCCAO}$ group.

We found that the level of MM-9 in the hippocampal CA1 region at $48 \mathrm{hr}$ after BCCAO did not differ. The level of MMP-9 in the hippocampal CA1 region was increased after BCCAO. In contrast, treadmill exercise decreased the expression of MMP-9 in the hippocampal CA1 region in the BCCAO group.

\section{DISCUSSION}

Learning and memory are impaired following chronic cerebral hypoperfusion induced by BCCAO in rats (Cechetti et al., 2012; Farkas et al., 2007). Treadmill exercise significantly ameliorated the spatial navigation performance of rats in the Morris water maze following chronic cerebral hypoperfusion (Lee et al., 2018b). The results of the present study demonstrate that preischemic treadmill exercise may attenuate short-term memory disturbance following chronic cerebral hypoperfusion.

Cerebral ischemia causes degradation of the basal lamina of microvessels and produces proteases known to degrade the extracellular matrix constituents (Fukuda et al., 2004). Cerebral hypoperfusion induced microvascular injury in the cortex and hippocampus, in contrast, treadmill exercise improved microvascular damage (Lee et al., 2017). The results of the present study demonstrated that preischemic treadmill exercise prevents damage to microvessels in the hippocampal CA1 region at 3 weeks after BC$\mathrm{CAO}$. These results indicate that preischemic treadmill exercise prevents microvascular injury in the hippocampus following chronic cerebral hypoperfusion.

Cerebral ischemia is a major contributor to BBB disruption via tight junction degradation (Liu et al., 2009; Yang et al., 2007). Early exercise significantly inhibited the ischemia-induced reduc- tion of tight junction (Zhang et al., 2013). Treadmill exercise attenuated the loss of ZO-1 in the brain after BCCAO (Lee et al., 2017). In the present study, at 3 weeks post-injury, the level of ZO-1 in the hippocampus was decreased after BCCAO. In contrast, preischemic treadmill exercise increased the expression of ZO-1 in the hippocampus after BCCAO. These findings suggest that preischemic treadmill exercise may offer protective effects on BBB disruption induced by chronic cerebral hypoperfusion.

Degradation of tight junction of the BBB is related to an increase in MMPs activity. Following transient focal ischemia, MMP-9 knock-out mice significantly reduced BBB disruption, which correlated with the degradation of ZO-1 compared to wildtype mice (Asahi et al., 2001). Preischemic exercise ameliorates BBB dysfunction in stroke by reducing MMP-9 expression (Guo et al., 2008). Treadmill exercise ameliorated the overexpression of MMP-9 after BCCAO (Lee et al., 2017). In the present study, preischemic treadmill exercise could significantly suppress the activation of MMP-9 in the brain following chronic cerebral hypoperfusion. Based on these findings, our results indicate that preischemic treadmill exercise may provide protective effects on BBB disruption from overexpression of MMP-9 induced by chronic cerebral hypoperfusion.

Preischemic treadmill exercise may ameliorate shot-term memory impairment and prevent microvascular injury in the hippocampus following chronic cerebral hypoperfusion. Therefore, preconditioning treadmill exercise might be used as a therapeutic strategy for the prevention of stroke in patients.

\section{CONFLICT OF INTEREST}

No potential conflict of interest relevant to this article was reported.

\section{ACKNOWLEDGMENTS}

This study was supported by the Ministry of Education of the Republic of Korea and the National Research Foundation of Korea (NRF-2016S1A5B5A01022873).

\section{REFERENCES}

Aboutaleb N, Shamsaei N, Khaksari M, Erfani S, Rajabi H, Nikbakht F. Pre-ischemic exercise reduces apoptosis in hippocampal CA3 cells after cerebral ischemia by modulation of the Bax/Bcl-2 proteins ratio and prevention of caspase-3 activation. J Physiol Sci 2015;65:435-443. 
Asahi M, Wang X, Mori T, Sumii T, Jung JC, Moskowitz MA, Fini ME, Lo EH. Effects of matrix metalloproteinase-9 gene knock-out on the proteolysis of blood-brain barrier and white matter components after cerebral ischemia. J Neurosci 2001;21:7724-7732.

Cechetti F, Worm PV, Elsner VR, Bertoldi K, Sanches E, Ben J, Siqueira IR, Netto CA. Forced treadmill exercise prevents oxidative stress and memory deficits following chronic cerebral hypoperfusion in the rat. Neurobiol Learn Mem 2012;97:90-96.

Cho JW, Jung SY, Kim DY, Chung YR, Choi HH, Jeon JW, Han JH. PI3KAkt-Wnt pathway is implicated in exercise-induced improvement of short-term memory in cerebral palsy rats. Int Neurourol J 2018;22 (Suppl3):S156-164.

Farkas E, Luiten PG, Bari F. Permanent, bilateral common carotid artery occlusion in the rat: a model for chronic cerebral hypoperfusion-related neurodegenerative diseases. Brain Res Rev 2007;54:162-180.

Feng S, Cen J, Huang Y, Shen H, Yao L, Wang Y, Chen Z. Matrix metalloproteinase- 2 and -9 secreted by leukemic cells increase the permeability of blood-brain barrier by disrupting tight junction proteins. PLoS One 2011;6:e20599.

Fukuda S, Fini CA, Mabuchi T, Koziol JA, Eggleston LL Jr, del Zoppo GJ. Focal cerebral ischemia induces active proteases that degrade microvascular matrix. Stroke 2004;35:998-1004.

Guo M, Cox B, Mahale S, Davis W, Carranza A, Hayes K, Sprague S, Jimenez D, Ding Y. Pre-ischemic exercise reduces matrix metalloproteinase-9 expression and ameliorates blood-brain barrier dysfunction in stroke. Neuroscience 2008;151:340-351.

Kumaran D, Udayabanu M, Kumar M, Aneja R, Katyal A. Involvement of angiotensin converting enzyme in cerebral hypoperfusion induced anterograde memory impairment and cholinergic dysfunction in rats. Neuroscience 2008;155:626-639.

Lee JM, Ji ES, Kim TW, Kim CJ, Shin MS, Lim BV, Chung YR, Cho YS. Treadmill exercise improves memory function by inhibiting hippocampal apoptosis in pilocarpine-induced epileptic rats. J Exerc Rehabil 2018a;14:713-723.

Lee JM, Kim CJ, Park JM, Song MK, Kim YJ. Effect of treadmill exercise on spatial navigation impairment associated with cerebellar Purkinje cell loss following chronic cerebral hypoperfusion. Mol Med Rep 2018b;17:8121-8128.

Lee JM, Park JM, Song MK, Oh YJ, Kim CJ, Kim YJ. The ameliorative effects of exercise on cognitive impairment and white matter injury from blood-brain barrier disruption induced by chronic cerebral hypoperfusion in adolescent rats. Neurosci Lett 2017;638:83-89.

Liu W, Hendren J, Qin XJ, Shen J, Liu KJ. Normobaric hyperoxia attenu- ates early blood-brain barrier disruption by inhibiting MMP-9-mediated occludin degradation in focal cerebral ischemia. J Neurochem 2009;108:811-820.

Moon EJ, Ko IG, Kim SE, Jin JJ, Hwang L, Kim CJ, An H, Lee BJ, Yi JW. Dexmedetomidine ameliorates sleep deprivation-induced depressive behaviors in mice. Int Neurourol J 2018;22(Suppl 3):S139-146.

O'Brien JT, Erkinjuntti T, Reisberg B, Roman G, Sawada T, Pantoni L, Bowler JV, Ballard C, DeCarli C, Gorelick PB, Rockwood K, Burns A, Gauthier S, DeKosky ST. Vascular cognitive impairment. Lancet Neurol 2003;2:89-98.

Sandoval KE, Witt KA. Blood-brain barrier tight junction permeability and ischemic stroke. Neurobiol Dis 2008;32:200-219.

Sim YJ, Kim SS, Kim JY, Shin MS, Kim CJ. Treadmill exercise improves short-term memory by suppressing ischemia-induced apoptosis of neuronal cells in gerbils. Neurosci Lett 2004;372:256-261.

Snyder HM, Corriveau RA, Craft S, Faber JE, Greenberg SM, Knopman D, Lamb BT, Montine TJ, Nedergaard M, Schaffer CB, Schneider JA, Wellington C, Wilcock DM, Zipfel GJ, Zlokovic B, Bain LJ, Bosetti F, Galis ZS, Koroshetz W, Carrillo MC. Vascular contributions to cognitive impairment and dementia including Alzheimer's disease. Alzheimers Dement 2015;11:710-717.

Tsuji K, Aoki T, Tejima E, Arai K, Lee SR, Atochin DN, Huang PL, Wang X, Montaner J, Lo EH. Tissue plasminogen activator promotes matrix metalloproteinase-9 upregulation after focal cerebral ischemia. Stroke 2005;36:1954-1959.

Xia YP, He QW, Li YN, Chen SC, Huang M, Wang Y, Gao Y, Huang Y, Wang MD, Mao L, Hu B. Recombinant human sonic hedgehog protein regulates the expression of $\mathrm{ZO}-1$ and occludin by activating angiopoietin-1 in stroke damage. PLoS One 2013;8:e68891.

Yang Y, Estrada EY, Thompson JF, Liu W, Rosenberg GA. Matrix metalloproteinase-mediated disruption of tight junction proteins in cerebral vessels is reversed by synthetic matrix metalloproteinase inhibitor in focal ischemia in rat. J Cereb Blood Flow Metab 2007;27:697-709.

Zhang GL, Deng JP, Wang BH, Zhao ZW, Li J, Gao L, Liu BL, Xong JR, Guo XD, Yan ZQ, Gao GD. Gypenosides improve cognitive impairment induced by chronic cerebral hypoperfusion in rats by suppressing oxidative stress and astrocytic activation. Behav Pharmacol 2011; 22:633-644.

Zhang Y, Zhang P, Shen X, Tian S, Wu Y, Zhu Y, Jia J, Wu J, Hu Y. Early exercise protects the blood-brain barrier from ischemic brain injury via the regulation of MMP-9 and occludin in rats. Int J Mol Sci 2013;14: 11096-11112. 